\title{
The shape of the Galactic halo with Gaia DR2 RR Lyrae. Anatomy of an ancient major merger
}

\author{
Giuliano Iorio ${ }^{1 \star}$ and Vasily Belokurov ${ }^{1,2} \dagger$ \\ ${ }^{1}$ Institute of Astronomy, University of Cambridge, Madingley Road, Cambridge CB3 OHA, UK \\ ${ }^{2}$ Centre for Computational Astrophysics, Flatiron Institute, 162 5th Avenue, New York, NY 10010, USA
}

Accepted XXX. Received YYY; in original form ZZZ

\begin{abstract}
We use the Gaia DR2 RR Lyrae sample to gain an uninterrupted view of the Galactic stellar halo. We dissect the available volume in slices parallel to the Milky Way's disc to show that within $\sim 30 \mathrm{kpc}$ from the Galactic centre the halo is triaxial, with the longest axis misaligned by $\sim 70^{\circ}$ with respect to the Galactic $x$-axis. This anatomical procedure exposes two large diffuse over-densities aligned with the semimajor axis of the halo: the Hercules-Aquila Cloud and the Virgo Over-density. We reveal the kinematics of the entire inner halo by mapping out the amplitudes and directions of the RR Lyrae proper motions. These are then compared to simple models with different anisotropies to demonstrate that the inner halo is dominated by stars on highly eccentric orbits. We interpret the shape of the density and the kinematics of the Gaia DR2 RR Lyrae as evidence in favour of a scenario in which the bulk of the halo was deposited in a single massive merger event.
\end{abstract}

Key words: galaxies: individual (Milky Way) - Galaxy: structure - Galaxy: stellar content - Galaxy: stellar halo - stars: (RR Lyrae) - Galaxy: kinematics

\section{INTRODUCTION}

In $\Lambda$ CDM Cosmology, Dark Matter halos are rarely spherical, their shapes controlled by the environment and the accretion history (Frenk et al. 1988; Dubinski \& Carlberg 1991; Warren et al. 1992; Colberg et al. 1999; Allgood et al. 2006; Bett et al. 2007; Hahn et al. 2007). At the early stages of the halo assembly, the shape is typically prolate and aligned with the narrow filaments, via which the mass is supplied, but with passing of time, halos can become triaxial or even oblate, as the feeding filaments swell and the direction of accretion changes (e.g. Cole \& Lacey 1996; Tormen 1997; Altay et al. 2006; Vera-Ciro et al. 2011; Libeskind et al. 2013). This metamorphosis does not necessarily imply that the memory of the early halo configuration is completely erased. Instead, at redshift zero, the history of the Dark Matter halo evolution may be deciphered by studying how its shape changes with Galactocentric radius (e.g. Hayashi et al. 2007; VeraCiro et al. 2011). Note however, that inclusion of baryons (see e.g. Kazantzidis et al. 2004; Gnedin et al. 2004; Debattista et al. 2008; Abadi et al. 2010) or adaptation of a different Dark Matter model (e.g. Avila-Reese et al. 2001;

^ giorio@ast.cam.ac.uk

$\dagger$ vasily@ast.cam.ac.uk
Davé et al. 2001; Mayer et al. 2002; Peter et al. 2013) can affect the details of some of the above calculations.

While the behavior of Dark Matter halos shows several coherent trends, stellar halos appear to display a wider diversity, linked to the strong suppression of star formation in low-mass Dark Matter clumps (see e.g. Bullock \& Johnston 2005; Cooper et al. 2010). One of the important corollaries of the above stochasticity is the expectation that the bulk of the (accreted) stellar halo of a Milky Way-like galaxy is contributed by a small number of massive dwarf galaxies (see e.g. De Lucia \& Helmi 2008; Deason et al. 2013). The picture therefore emerges in which the most massive halo progenitors not only can define the shape of the stellar halo (see Deason et al. 2013) but also set its overall metallicity (Deason et al. 2016; D'Souza \& Bell 2018). It is difficult to produce stellar halos without invoking (at least some of) the processes that lead to formation of stars. The inclusion of baryonic physics tends to alter the shapes of the resulting stellar halos significantly. For examples, the inner portions of the stellar halos built up with semi-analytic machinery are often prolate (see e.g. Cooper et al. 2010), while hydro-dynamical simulations deliver mostly oblate shapes (e.g. Monachesi et al. 2018). The prevalence of the oblate shapes in the simulated stellar halos is sometimes linked to the significant contribution of so-called in-situ component 
(Benson et al. 2004; Zolotov et al. 2009; McCarthy et al. 2012; Tissera et al. 2013; Cooper et al. 2015).

Early attempts to gauge the properties of the Milky Way's stellar halo had to rely on the small number of tracers and/or sparse sky coverage (Preston et al. 1991; Reid \& Majewski 1993; Sluis \& Arnold 1998; Morrison et al. 2000; Siegel et al. 2002). Recently, thanks to the availability of wide-area deep imaging data, the shape of the Milky Way's stellar halo has been the focus of many studies (e.g. Newberg \& Yanny 2006; Bell et al. 2008; Jurić et al. 2008; Sesar et al. 2011; Deason et al. 2011; Xue et al. 2015; Iorio et al. 2018). While surveys like the SDSS (see Alam et al. 2015) do provide a much broader view of the halo (see e.g. Carollo et al. 2007, 2010), large swathes of the sky are still missing, leaving portions of the inner Galaxy unmapped. Most recently, the Gaia mission (see Gaia Collaboration et al. 2016) has provided the first all-sky view of the Galactic halo. By combining the variability statistics from Gaia DR1 with the color information from Gaia and 2MASS, Iorio et al. (2018) built a sample of $\sim 22,000 \mathrm{RR}$ Lyrae covering most of the celestial sphere, except for narrow regions close to the Galactic plane. Using these old and metal-poor pulsating stars and taking advantage of largely un-interrupted view of the Galaxy, they were able to test a wide range of stellar halo models.

In this Paper, we aim to use the Gaia DR2 RR Lyrae stars to get both closer to the centre of the Milky Way and to go further beyond the reach of the Iorio et al. (2018) analysis by linking the RR Lyrae density evolution with their kinematics. Our study is motivated by the recent discovery of tidal debris from what appears to be an ancient major merger event (Belokurov et al. 2018b; Myeong et al. 2018b; Helmi et al. 2018) Identified first in the Solar neighborhood, this debris cloud, sometimes referred to as the "Gaia Sausage" has recently been shown to dominate the Galactic stellar halo, stretching from regions in the Milky Way's bulge to near and past the halo's break radius around 20-30 kpc (see Deason et al. 2018; Simion et al. 2018; Lancaster et al. 2018). Additional evidence has been found in the studies of the detailed chemical abundances of the nearby halo stars (see Hayes et al. 2018; Haywood et al. 2018; Mackereth et al. 2018). While many pieces of the "Gaia Sausage" have already been reported in the literature, here we attempt to provide the first comprehensive map of this largest halo sub-structure. The kinematic portion of our study is complementary to the work of Wegg et al. (2018), who recently used a sample of PanSTARRS1 RR Lyrae stars to constrain the shape of the inner portion of the Galactic gravitational potential.

This Paper is organized as follows. Section 2 describes the construction of the clean sample of Gaia DR2 RR Lyrae stars. In Section 3 we show how these objects can be used to slice the Galactic halo to reveal the remnant of a large dwarf galaxy buried close to the Milky Way's centre. In Section 4 we complement the spatial analysis with an all-sky RR Lyrae kinematic map and we discuss the implications of our discovery. Finally, we summarise the conclusions of this work in Section 5.

\section{THE Gaia DR2 RR LYRAE SAMPLE}

\subsection{RR Lyrae stars in Gaia DR2}

The Gaia DR2 catalogue (Gaia Collaboration et al. 2018a, 2016) contains 550,737 variable stars, among which more than 200,000 are classified as RR Lyrae (RRL) stars (Holl et al. 2018). In the Gaia data, the RRL stars are spread across separate catalogues: some are in the tables reporting the results of the general variability analysis (e.g. vari_classifier_result and vari_time_series_statistics); others can be found in the SOS (Specific Object Studies, Clementini et al. 2018) table vari_rrlyrae. For further details on the variable stars classification and analysis in Gaia DR2 please see Holl et al. (2018). About half of the RRL stars are shared between the SOS and the other tables, thus in order to merge all classified RRL stars into one final sample, we joined the table vari_classifier_result and the table vari_rrlyrae using the source_id unique identifier of each star. The final sample contains 228,853 RRL stars ( 77\% RRab, 21\% RRc and $\sim 2 \%$ RRd). Finally, this table containing all of the Gaia DR2 RRL stars, was merged with the main gaia_source catalogue, thus complementing the RRL positions with photometry and astrometry.

The top-left panel of Fig. 1 shows the distribution of the RRL stars in our sample as a function of the Galactic coordinates $(\ell, b)$. A number of genuine as well as spurious objects stand out against the underlying diffuse distribution of stars. These include the Magellanic Clouds, the Sagittarius dwarf galaxy and its tails, a number of other dwarf spheroidal satellites and globular clusters, together with over-densities close to the Galactic plane. In order to study the broad-brush properties of the stellar halo, we first attempt to clean the sample and remove RRL stars belonging to the most obvious compact structures, including both bona fide satellites as well as data artefacts and contaminants. The following section describes in detail the selection cuts applied to clean the Gaia DR2 RRL sample.

\subsection{Cleaning}

Artefacts and contaminants. The percentage of contaminants in the Gaia DR2 RRL sample has been estimated in Holl et al. (2018) and in Clementini et al. (2018) using auxiliary catalogues. They found a low level of contaminants (5\%$15 \%$ ) except in the region of the bulge and in the area close to the Galactic disc where the contamination level appears to increase up to many tens of per cent. The contaminants in these crowded fields are predominantly eclipsing binaries and blended sources, with a tiny number of spurious detections due to misclassified variable stars (Holl et al. 2018). To cull the majority of the likely contaminants we only keep objects that satisfy the following selection criteria:

- $A E N<0.25$,

- $B R E<1.5$,

- $E(B-V)<0.8$.

The cut on the astrometric_excess_noise, AEN, eliminates unresolved stellar binaries, blends and galaxies (see e.g. Koposov et al. 2017) as their astrometric behavior deviates from that of the single point sources (see Lindegren et al. 

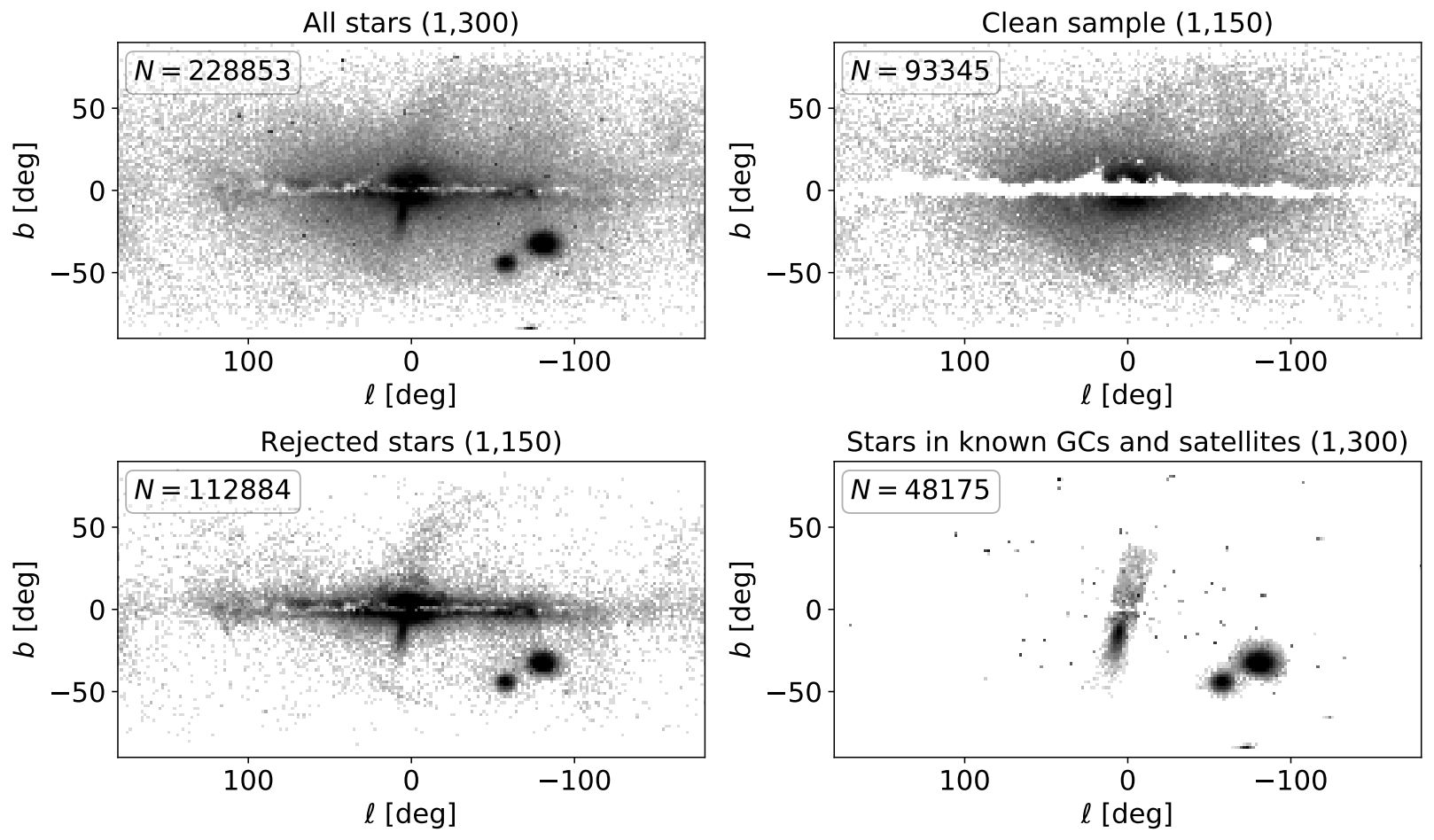

Figure 1. All-sky RR Lyrae density maps in Galactic coordinates $(\ell, b)$. Top-left: original sample of stars classified as RR Lyrae in Gaia DR2. Top-right: clean sample of RR Lyrae stars used in this work. Bottom-left: objects filtered from the original sample using a combination of photometric and astrometric selection cuts. Bottom-right: stars in the original sample belonging to known globular clusters, dwarf spheroidal satellites, the Sagittarius dwarf and a part of its stream and the Magellanic Clouds. The numbers in brackets in the title of each panel indicate the dynamic range of the grey-scale map; the number in the top-left corner indicates the total number of stars in each panel.

2012). The $p h o t \_b p \_r p \_e x c e s s \_f a c t o r, B R E$, represents the ratio between the combined flux in the Gaia $B P$ and $R P$ bands and the flux in the $G$ band, and thus by design is large for blended sources (see Evans et al. 2018). Finally, we remove stars in regions with high reddening, $E(B-V)$ (according to Schlegel et al. 1998), for which the dust extinction correction is likely unreliable (see Sec. 3.1).

Globular clusters and dwarf satellites. We have removed all stars within twice the half-light radius of all known globular clusters listed in Harris (2010). Concerning the dwarf spheroidal satellites, we excluded all stars within an angular distance of $0.5^{\circ}$ from the centre of Carina, Ursa Minor and Hercules, $0.3^{\circ}$ from Leo I and Leo II, and $1^{\circ}$ from Sculptor, Fornax, Draco and Sextans.

Sagittarius dwarf. First, we selected all stars with $\left|\tilde{B}-\tilde{B}_{\mathrm{Sgr}}\right|<10^{\circ}$ and $\left|\tilde{\Lambda}-\tilde{\Lambda}_{\mathrm{Sgr}}\right|<50^{\circ}$, where $\tilde{B}$ and $\tilde{\Lambda}$ are the latitude and longitude in the coordinate system aligned with the Sagittarius stream as defined in Belokurov et al. (2014) and $\tilde{B}_{\mathrm{Sgr}}=4.24^{\circ}$ and $\tilde{\Lambda}_{\mathrm{Sgr}}=-1.55^{\circ}$ represent the position of the Sagittarius dwarf. Then, among the selected objects, we got rid of all stars with a proper motion relative to Sagit-

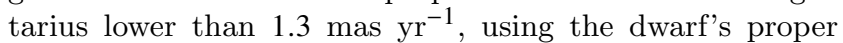
motion as reported in Gaia Collaboration et al. (2018b). These spatial and kinematical selection cuts appear to be effective in removing the Sagittarius dwarf and the initial portions of its tails, as demonstrated in Fig. 1. We have decided against extending the filter to the rest of the Sagit- tarius stream $\left(\left|\tilde{\Lambda}-\tilde{\Lambda}_{\mathrm{Sgr}}\right|>50^{\circ}\right)$ to avoid over-cleaning our sample at large Galactic latitudes.

Magellanic Clouds. In order to identify (and remove) the stars that belong to the Clouds, we selected all objects within an angular distance of $16^{\circ}\left(12^{\circ}\right)$ from the LMC (SMC). Of these, only the stars with proper motion relative to the LMC or to the SMC lower than 5 mas $\mathrm{yr}^{-1}$ with respect to the values reported in van der Marel \& Sahlmann (2016) were retained for further consideration. Given the expected $G$ magnitude of the RRL stars at the distance of the LMC and SMC (Belokurov et al. 2017), we required the Magellanic stars to have $18.5<G<20$ ( $G$ corrected for the extinction, see Sec. 3.1). Finally, in order to avoid the crowded central part of the Clouds, we selected all stars within $5^{\circ}$ from the LMC and SMC centre, independently of their proper motions and $G$ magnitudes. The stars that survived all of the selection cuts above were removed from our RRL sample.

The sky distribution of the likely artefacts discarded by the astrometric and the photometric cuts described above is shown in the bottom-left panel of Fig. 1. As expected, most of the contaminants are located close to the Galactic plane, while others can be found in the crowded regions of the Magellanic Clouds and the Sagittarius dwarf galaxy. The distribution of the (removed) stars projected to lie within known Galactic satellites (globular clusters, dwarf galaxies) is shown in the bottom right-panel. The stellar density dis- 
tribution in the final clean sample containing $~ 93,000$ RRL stars is shown in the top-right panel.

\section{GALACTIC HALO SHAPE WITH Gaia DR2 RR LYRAE}

\subsection{RR Lyrae distances}

In the analysis presented below, we use a left-handed Cartesian reference frame $(x, y, z)$ centred in the Galactic centre as defined in Iorio et al. (2018). In this reference frame, the Sun is at $(8,0,0) \mathrm{kpc}$. Despite the photometric variability, RRL stars have an almost constant absolute magnitude in the visual (see e.g. Catelan et al. 2004). Thus having measured the apparent magnitudes $G$ (corrected for the dust extinction), we can directly estimate the heliocentric distances as

$\log \left(\frac{D_{\odot}}{\mathrm{kpc}}\right)=\frac{G-M_{G}}{5}-2$

Before applying Eq. 1, we correct the observed apparent magnitudes for the dust extinction and estimate $M_{G}$ exploiting the stars in our sample with robust parallax measurements.

Dust extinction. The $G$ band absorption $A_{G}=$ $k_{G} E(B-V)$ for the RRab stars has been estimated in the SOS (Clementini et al. 2018) through the empirical relation derived by Piersimoni et al. (2002). We used these stars to directly estimate the extinction coefficient $k_{G}=A_{G} / E(B-V)$, where the reddening $E(B-V)$ is obtained from the maps of Schlegel et al. (1998). The derived $k_{G}$ distribution shows a narrow peak at $1<k_{G}<3.4$. We fit a Gaussian model to the measurements of stars inside this $k_{G}$ range taking into account the errors with the help of the extreme deconvolution technique (ED, Bovy Jo et al. 2011). The best-fit centroid is at $k_{G}=2.27$, i.e. a value slightly lower compared to that reported previously (e.g. Belokurov et al. 2017). To summarise, the observed apparent magnitudes of all stars in our sample have been corrected using $A_{G}=2.27 E(B-V)$.

Absolute magnitude in the $G$ band. Inverting Eq. 1 and using the parallax $\varpi$, the absolute magnitude can be estimated as

$M_{G}=G+5 \log \varpi-10$.

We selected 837 stars from the clean sample i) with robust estimates of the parallax $(\varpi / \delta \varpi>10)$ and ii) located in regions where the correction for dust extinction is almost negligible, i.e. $E(B-V)<0.1$. Before using Eq. 2, we corrected the parallaxes for the offset $\varpi=0.029$ reported in Lindegren et al. (2018) (see also Muraveva et al. 2018). The estimated $M_{G}$ distribution exhibits a narrow peak for $\left|M_{G}\right|<1.5$. As in the case of the reddening coefficient described above, the distribution was modeled as a Gaussian taking into account the errors in $\varpi$ using the ED machinery. The best-fit centroid is at $M_{G}=0.64$ and the best-fit dispersion is $\sigma_{M_{G}}=0.25$. We estimated the possible systematic effects repeating the analysis considering only the stars classified as RRab $\left(M_{G}=0.64\right)$ or $\operatorname{RRc}\left(M_{G}=0.60\right)$ in the SOS and considering only the metal poor $[\mathrm{Fe} / \mathrm{H}]<-1.0$ $\left(M_{G}=0.58\right)$ and the metal rich $[\mathrm{Fe} / \mathrm{H}]>-1.0\left(M_{G}=0.72\right)$
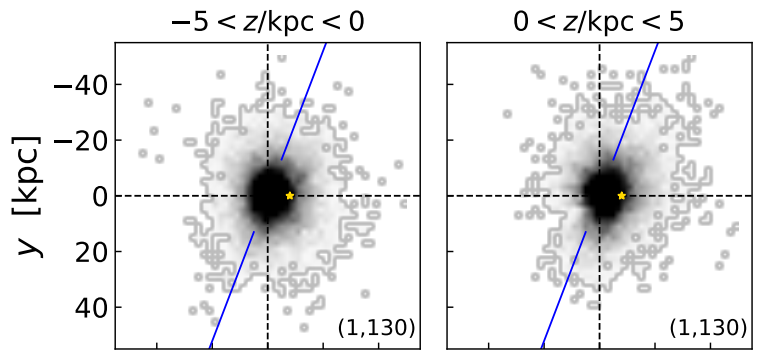

$-10<z / k p c<-5$

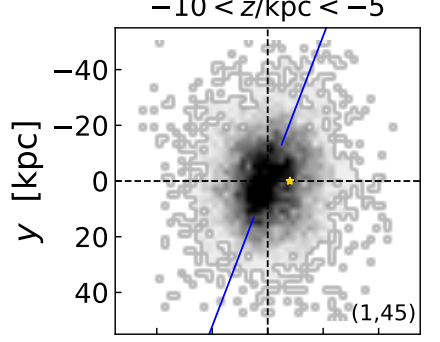

$5<z / k p c<10$

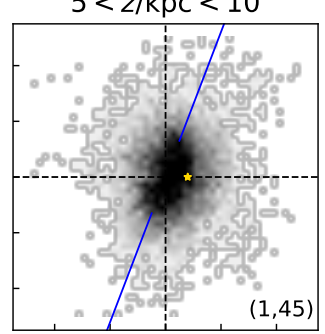

$10<z / \mathrm{kpc}<30$
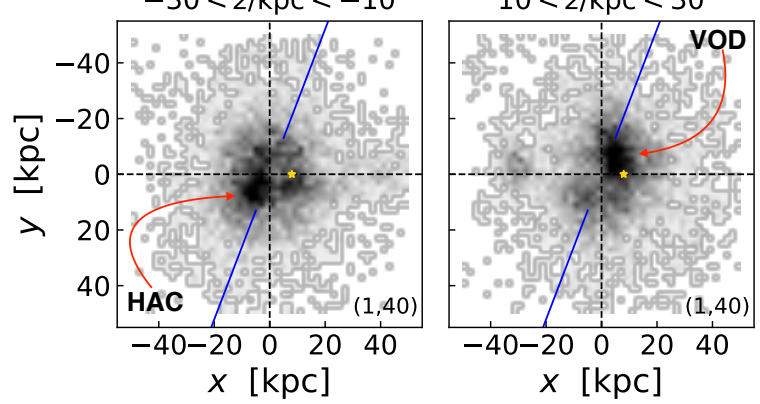

Figure 2. RR Lyrae density projected onto $x-y$ plane for three pairs of $z$ slabs. The two slabs in each pair are positioned symmetrically with respect to the Galactic plane. The $z$ range is reported in the title of each panel. Yellow star indicates the position of the Sun ( $x=8 \mathrm{kpc}, y=z=0 \mathrm{kpc})$. Dynamic range of the greyscale map is given in brackets in the bottom-right corner in each panel. Blue lines indicate the orientation of the major axis of the triaxial stellar halo model described in Iorio et al. (2018). The red arrows point out the location of the Hercules-Aquila Cloud (HAC) and the Virgo Over-Density (VOD).

stars $^{1}$. We also repeated the analysis not correcting for the parallax offset $\left(M_{G}=0.45\right)$ or using the distance estimated from the parallaxes in Bailer-Jones et al. (2018) $\left(M_{G}=0.66\right)$. Overall all the systematic differences are within the measured spread in $M_{G}$. For the rest of the paper, we consider $M_{G}=0.64$ for all the stars in our clean sample. Note that a difference in magnitude of 0.25 corresponds to an uncertainty in distance of $\sim 1 \mathrm{kpc}$ for a star at $10 \mathrm{kpc}$ and $\sim 4 \mathrm{kpc}$ for a star at $50 \mathrm{kpc}$. Using apparent magnitudes corrected for dust extinction and applying the assumed (constant) value of the absolute magnitude, we estimated the distance (Eq. 1 ) and the Galactocentric Cartesian $(x, y, z)$ coordinates of each RRL star in our clean sample.

1 We used the metallicities reported in the SOS catalogue, see Clementini et al. (2018) for further details 

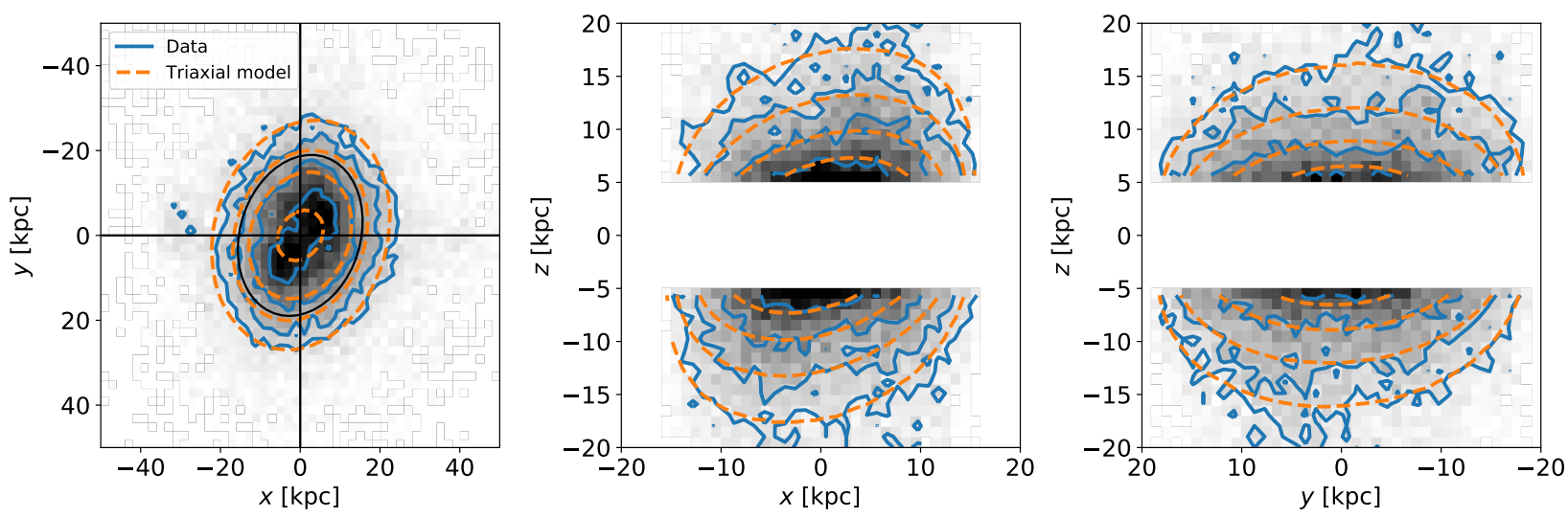

Figure 3. Three different projections of RR Lyrae density in the inner halo. Normalized star-counts $\left(N / N_{\text {tot }}\right)$ are shown projected onto the principal planes in Galactic coordinates. Only stars with $5<|z / \mathrm{kpc}|<30$ are included. The Gaia DR2 data (triaxial model) are shown as a greyscale map and blue contours (orange contours, see text for details). The contours are at $N / N_{\text {tot }}=(0.5,1,2,4) \times 10^{-4}$. Left-hand: $x-y$ plane, the black ellipse has a major-to-minor axial ratio of $p=1.3$ and the major axis $\left(a=19.5 \mathrm{kpc}\right.$ ) is tilted by $21^{\circ}$ with respect to the $y$-axis. Middle: $x-z$ plane density of stars within the ellipse shown in the left-hand panel; Right-hand: $y-z$ plane density of stars within the ellipse shown in the left-hand panel.

\subsection{Slicing the halo}

Fig. 2 shows the distribution of stars in the Galactic $x-y$ plane in different $z$-slices. Close to the Galactic disc $(0<$ $|z / \mathrm{kpc}|<5)$, the distribution of stars is symmetric with respect to the Galactic plane and most of the stars can be found within $10 \mathrm{kpc}$ from the Galactic centre. At the intermediate heights $(5<|z / \mathrm{kpc}|<10)$, the distribution preserves the semblance of symmetry with respect to the Galactic plane, but the bulk of the stars is now arranged within an elongated structure of roughly ellipsoidal shape. The major axis of this structure is tilted by about $70^{\circ}$ (anti-clockwise) with respect to the Galactic $x$-axis. At even larger heights $(10<|z / \mathrm{kpc}|<30)$, two over-dense regions are clearly visible: one above the Galactic plane in the quadrant with positive $x$ and negative $y$ and one below the Galactic plane, in the quadrant with negative $x$ and positive $y$. The structure above the Galactic plane is the Virgo Over-Density (VOD, Vivas et al. 2001; Newberg et al. 2002; Duffau et al. 2006; Jurić et al. 2008; Bonaca et al. 2012), while the one below is the Hercules-Aquila Cloud (HAC, Belokurov et al. 2007; Simion et al. 2014). Note that these two striking over-densities appear to lie at very similar distances from the Galactic centre.

Apart from the VOD and the HAC, other minor substructures can be seen in the last two $z$-slices: a circular overdensity of stars centred at $x \sim-30 \mathrm{kpc}$ above the Galactic plane and a feeble overdensity strip between $x \sim 15 \mathrm{kpc}$ and $x \sim 40 \mathrm{kpc}$ below the Galactic plane. Both of these are the remaining portions of the Sagittarius stream at large (in absolute values) Galactic latitudes (see Sec. 2). We stress that the residuals of the Sagittarius stream cannot be responsible of the elongated structure shown in Fig. 2, in fact, the signature of the stream runs almost exactly perpendicular to it, both above and below the Galactic plane. The possible random and systematic errors due to the assumption of a constant $M_{G}$ act akin to spatial smoothing (of the order of $1-4 \mathrm{kpc}$ ) in the distribution of stars shown in Fig. 2 . This smearing effect should not have any preferential direction, thus we expect that, in the ideal case of perfect distance measurements, the elongated density structure ought to emerge even more clearly. In conclusion, we believe that the shape and the orientation of the RRL distribution shown in Fig. 2 represent faithfully the intrinsic properties of the inner Galactic stellar halo.

The presence of a similar elongated density distribution of stars in the halo was claimed previously by Newberg \& Yanny (2006) and, most recently by Iorio et al. (2018) who used shallower $(G<17.1)$ sample of RRL star candidates from Gaia DR1. The best halo model reported in Iorio et al. (2018) is triaxial with a flattening along the direction normal to the Galactic disc and the major axis lying in the Galactic plane. The direction of the major axis is overplotted in the $x-y$ density maps in Fig. 2. Evidently, the ellipsoid's orientation inferred by Iorio et al. (2018) is compatible with the elongation of the RRL distribution at intermediate heights and is aligned with the direction connecting the VOD and the HAC structures.

Fig. 3 presents a detailed comparison between the data and the model similar to that introduced in Iorio et al. (2018). The left-hand panel of the Figure shows the $x-y$ projection of the distribution of the inner halo stars with $5<|z / \mathrm{kpc}|<30$. The middle (giving the $x-z$ view) and right-hand $(y-z)$ panels only concern the 21,269 stars within the cylinder with $5<|z / \mathrm{kpc}|<30$ and the base shown as a black ellipse in the left panel of the Figure. The stellar distributions in the last two planes are clearly different. In particular, the iso-density contours in the $x-z$ plane are not aligned with the Galactic axes. It is interesting to explore whether the distribution of stars whose projections on the three principal planes shown in Fig. 3 can be reproduced by a smooth triaxial halo model. For this purpose, we over-plot the iso-density contours of the best-fit model labeled SPL$\mathrm{TR}^{q v}$ in Iorio et al. (2018). The original model is triaxial, with the major axis lying in the Galactic plane and the minor axis pointing along the Galactic $\mathrm{z}$-axis. The ratio of major to intermediate axis is 1.27 and the flattening along the vertical direction (i.e. minor axis) decreases with the elliptical radius (i.e. the halo becomes more spherical). In order to describe better the tilted distribution of stars in $x-z$ plane, the model 
was modified by adding a rotation around the major axis of $\beta=20^{\circ}$ (see Iorio et al. 2018 for a detailed description of the tilt angles formalism). Curiously, this model is similar to the best-fit triaxial halo model found in Newberg \& Yanny (2006), except for the variation of the vertical flattening. The match between the data and the model is impressive, especially considering that the fit was performed exploiting only a limited portion of the halo's volume (Newberg \& Yanny 2006; Iorio et al. 2018). Note, however, that according to the left-hand panel in Fig. 3, it is not possible to reproduce the double peak in the projection of the density onto the $x-y$ plane. These two off-centered over-densities correspond to the VOD and the HAC.

Overall, the picture that emerges from Fig. 2 and Fig. 3 is that of a triaxial stellar halo, misaligned with respect to the $x$-axis and possibly misaligned with respect to the Galactic plane. The latter statement is less certain due to the presence of two large stellar over-densities, the VOD (in the Galactic North) and the HAC (in the South). These large and diffuse Clouds nonetheless appear to be connected to the stellar halo's ellipsoid, being arranged approximately along its major axis.

\section{ANATOMY OF AN ANCIENT MAJOR MERGER}

Confronted with the image of the stretched, likely triaxial inner stellar halo as revealed in Figures 2 and 3, it is difficult to envisage a scenario in which such a structure emerges naturally as a result of multiple randomly oriented accretion events. On the other hand, major mergers tend to produce exactly such a configuration of debris - an ellipsoid elongated in the direction of the collision (e.g. Moore et al. 2004; Cooper et al. 2010). Even if the post-merger halo is nearly axi-symmetric e.g. prolate, the shape can evolve with time, under the influence of the growing baryonic component (see e.g. Debattista et al. 2008; Bryan et al. 2013; Tomassetti et al. 2016).

Coincidentally, weighty evidence for an old accretion event of a massive satellite on a low angular momentum orbit has recently been uncovered in the Milky Way (see Belokurov et al. 2018b; Myeong et al. 2018b; Helmi et al. 2018; Haywood et al. 2018). The give-away sign that the object that merged with the Galaxy was massive is the metallicity distribution of its tidal debris which reaches $[\mathrm{Fe} / \mathrm{H}] \sim-1$ (or perhaps, even higher). The independent confirmation of the progenitor's high mass comes from the discovery of a large number of Globular Clusters likely associated with the event (e.g. Myeong et al. 2018a; Kruijssen et al. 2018). The geometry of the impact can be gleaned from the orbital anisotropy of the accreted stars: typical values of $\beta \sim 0.9$ (the extreme stretching of the velocity ellipsoid gained this halo component the name "Gaia Sausage") correspond to eccentricities not too far from 1 . This head-on collision appears to have sprayed stars all over the Milky Way, dominating the stellar halo out to $\sim 30 \mathrm{kpc}$ as evidenced from the recent studies of the orbital properties of a variety of halo tracers across a wide range of Galactocentric distances (Bird et al. 2018; Deason et al. 2018; Lancaster et al. 2018). Finally, using a sample of RRL stars with radial velocities, Simion et al. (2018) found that the HAC and VOD can also be linked to this event, representing some of the less mixed portions of its tidal debris. In the face of the evidence above, the obvious question is whether some or most of the stars in the triaxial structure revealed here (see Sec. 3.2) belong to the "Gaia Sausage".

With this conjecture in mind, we set out to explore the kinematics of the RRL stars in the inner portions of the Milky Way's halo.

\subsection{RR Lyrae kinematics}

Unfortunately, with only the proper motions measurements at our disposal, we cannot directly study the orbital properties of the RRL stars in our sample. Recently Wegg et al. (2018) circumvented this limitation using a sample of PanSTARRS1 RRL stars in Gaia DR2 and marginalising over the unknown line-of-sight velocity component. In this work, we decided to use a simpler, but nonetheless effective and illuminating approach. In particular, we compare the observed proper motions distributions to the predictions of several simplified halo models. More precisely, we generate mock proper motions values for the stars in our sample assuming i) complete orbital isotropy, ii) high radial anisotropy (similar to the "Gaia Sausage") as well as iii) strong tangential anisotropy. The model velocity distributions in Galactocentric spherical coordinates are shown in the top-left panel of Fig. 4. The isotropic model is composed of Gaussian velocity distributions with $\sigma_{\theta}=\sigma_{\phi}=\sigma_{\mathrm{r}}=120 \mathrm{~km} \mathrm{~s}^{-1}$. The radially anisotropic model is inspired by that used in Necib et al. (2018) and Lancaster et al. (2018). Namely, the $V_{\mathrm{r}}$ distribution contains two Gaussians with peaks at $V_{\mathrm{r}}= \pm 160 \mathrm{~km} \mathrm{~s}^{-1}$ and $\sigma_{\mathrm{r}}=130 \mathrm{~km} \mathrm{~s}^{-1}$, while the $V_{\theta}$ and $V_{\phi}$ distribution are single Gaussians centred in 0 and with $\sigma_{\theta}=\sigma_{\phi}=30 \mathrm{~km} \mathrm{~s}^{-1}$. Therefore, this model corresponds to the extreme orbital anisotropy of $\beta \sim 0.96$, similar to that observed for the "Gaia Sausage" stars (Belokurov et al. 2017; Myeong et al. 2018b; Simion et al. 2018). The tangentially biased model uses Gaussian velocity distributions with $\sigma_{\theta}=\sigma_{\phi}=100 \mathrm{~km} \mathrm{~s}^{-1}$ and $\sigma_{\mathrm{r}}=40 \mathrm{~km} \mathrm{~s}^{-1}$, these values are arbitrary and have been set to reproduce a strong tangential anisotropy $(\beta=-5.25)$ maintaining roughly the same velocity range of the isotropic and radially anisotropic models (see Fig. 4). Note, however, that $\beta$ of our tangentially anisotropic model is somewhat lower (signifying a stronger anisotropy) than the lowest anisotropy estimates based on stellar tracers in the Milky Way stellar halo. (see e.g. Kafle et al. 2012; King et al. 2015). In the analysis that follows, we focus only on the $\sim 21,000$ stars belonging to the triaxial structure highlighted in Sec. 3.2, i.e. those within the region with $5<|z / \mathrm{kpc}|<30$ and inside the black ellipse shown in the left-hand panel of Fig. 3. In practice, for each RRL star in our sample, we draw $\left(V_{\mathrm{r}}, V_{\theta}, V_{\phi}\right)$ randomly from the distributions discussed above. Then, given the fully defined positions and velocity vectors, we derive the model proper motions $\left(\mu_{\ell}, \mu_{b}\right)$. The final model predictions (see below) are obtained by averaging over 10,000 model realisations.

Armed with the halo models, we compress the kinematic information into two simple plots. First, as shown in Fig. 4 we compare the $1-\mathrm{D}$ distributions of $\mu_{\ell}$ and $\mu_{b}$ across the entire sample of stars considered. From isotropic, through tangentially to radially anisotropic models, the proper motion 

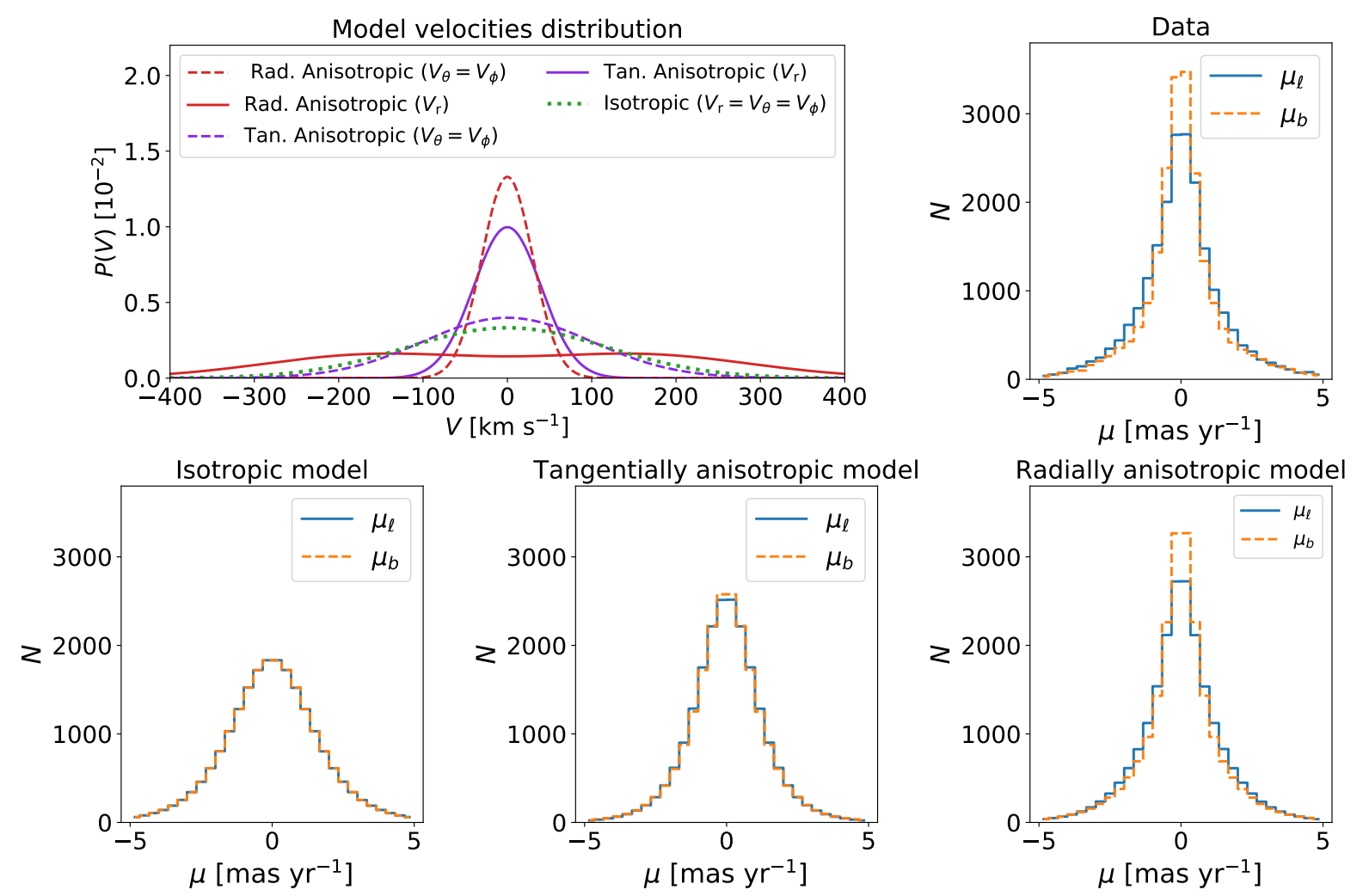

Figure 4. Galactic proper motion distributions in the Gaia DR2 RR Lyrae sample and 3 different halo models. Isotropic (bottom-left panel), tangentially anisotropic (bottom-middle panel) and radially anisotropic (bottom-right panel) models are shown. These can be compared to the observed $\left(\mu_{\ell}, \mu_{b}\right)$ distributions given in the top-right panel. Only stars in the clean sample with $5<|z / \mathrm{kpc}|<30$ and located within the ellipse shown in the left-hand panel of Fig. 3 are considered $(N \sim 21,000)$. The model proper motions are obtained by assigning to each star in our sample a Galactocentric spherical velocity $\left(V_{\mathrm{r}}, V_{\theta}, V_{\phi}\right)$ randomly drawn from the corresponding distributions shown in the top-left panel. The model histograms are acquired by taking the averaging deviation of 10,000 model realisations. The dispersion of this realisation set is negligible (less than $1 \%$ at the histogram peaks) and it is not shown. All proper motions have been corrected for the Solar reflex motion (Schönrich 2012).

distributions become narrower. The thinnest peak belongs to the distribution predicted by the radially anisotropic model, which also possesses sizeable wings. For the isotropic and the tangential case, the shapes of the $\mu_{\ell}$ and the $\mu_{b}$ histograms are indistinguishable. However, for the radially biased model, the distributions of the two proper motion components are clearly different: the $\mu_{b}$ histogram is more peaked with respect to that of $\mu_{\ell}$. Most importantly, there is a strikingly good match between the radially biased model and the data shown in the top-right panel of Fig. 4.

Fig. 5 extends the comparison from 1-D to 2-D, i.e. demonstrates the behavior of the three models and the data as a function of the position on the sky. Here we show the evolution of the Pearson correlation coefficient $\rho_{\mu_{\ell}, \mu_{b}}{ }^{2}$ in bins of Galactic coordinates. The thus calculated correlation can be interpreted as the characteristic direction of motion on the plane of the sky. No preferred direction is expected for the isotropic model as confirmed in the top-left panel of Fig. 5. In spherical polars, the velocity ellipsoids of the tangentially and radially biased models are rotated by $\pi / 2$

${ }^{2} \rho_{\mu_{\ell}, \mu_{b}}=\operatorname{cov}\left(\mu_{\ell}, \mu_{b}\right) / \sigma_{\mu_{\ell}} \sigma_{\mu_{b}}$, where $\operatorname{cov}\left(\mu_{\ell}, \mu_{b}\right)$ is the proper motions covariance and $\sigma_{\mu_{\ell}}$ and $\sigma_{\mu_{b}}$ are the proper motion standard deviations. with respect to each other. As the top-right and the bottomleft panels demonstrate, even in the absence of the third velocity component, the stretching and the orientation of the velocity ellipsoid can be deduced from its projection on the sky (for reasonably nearby stars as those considered here). In the presence of strong orbital anisotropy, the proper motions are heavily correlated. The sign of the correlation depends on sky position and changes every $90^{\circ}$ both in $\ell$ and $b$ creating the characteristic "red-blue" patterns visible in the top-right and bottom-left panels of the Fig. 5. Without any doubt, the observed pattern of the RRL motion shown in the bottomright panel matches that of the radially-anisotropic model (bottom-left).

We stress that the predictions of our simple kinematical models have converged, i.e. they do not change when the number of realisations is increased. In particular, the dispersion of the model representations is negligible in Fig. 4 (less than $1 \%$ at the histograms peaks) and the mean dispersion in the correlation maps in Fig. $5\left(\sigma_{\rho_{\mu_{\ell}, \mu_{\mathrm{b}}}} \sim 0.25\right)$ is compatible with the level of random signal oscillation shown in the data. In fact, even when using a single realisation the relevant features shown in Fig. 4 and Fig. 5 are still evident, although the overall proper motion distributions and correlation maps appear noisier. 


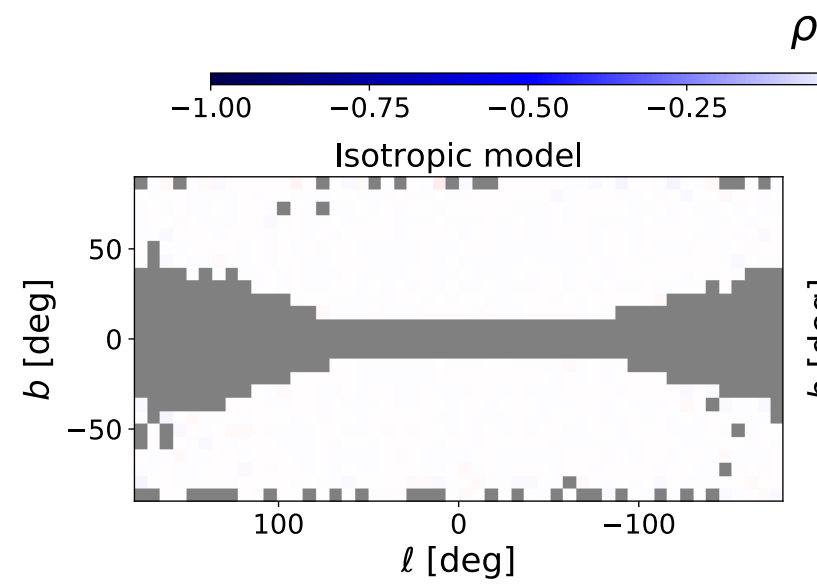

$\rho_{\mu_{\ell}, \mu_{b}}$
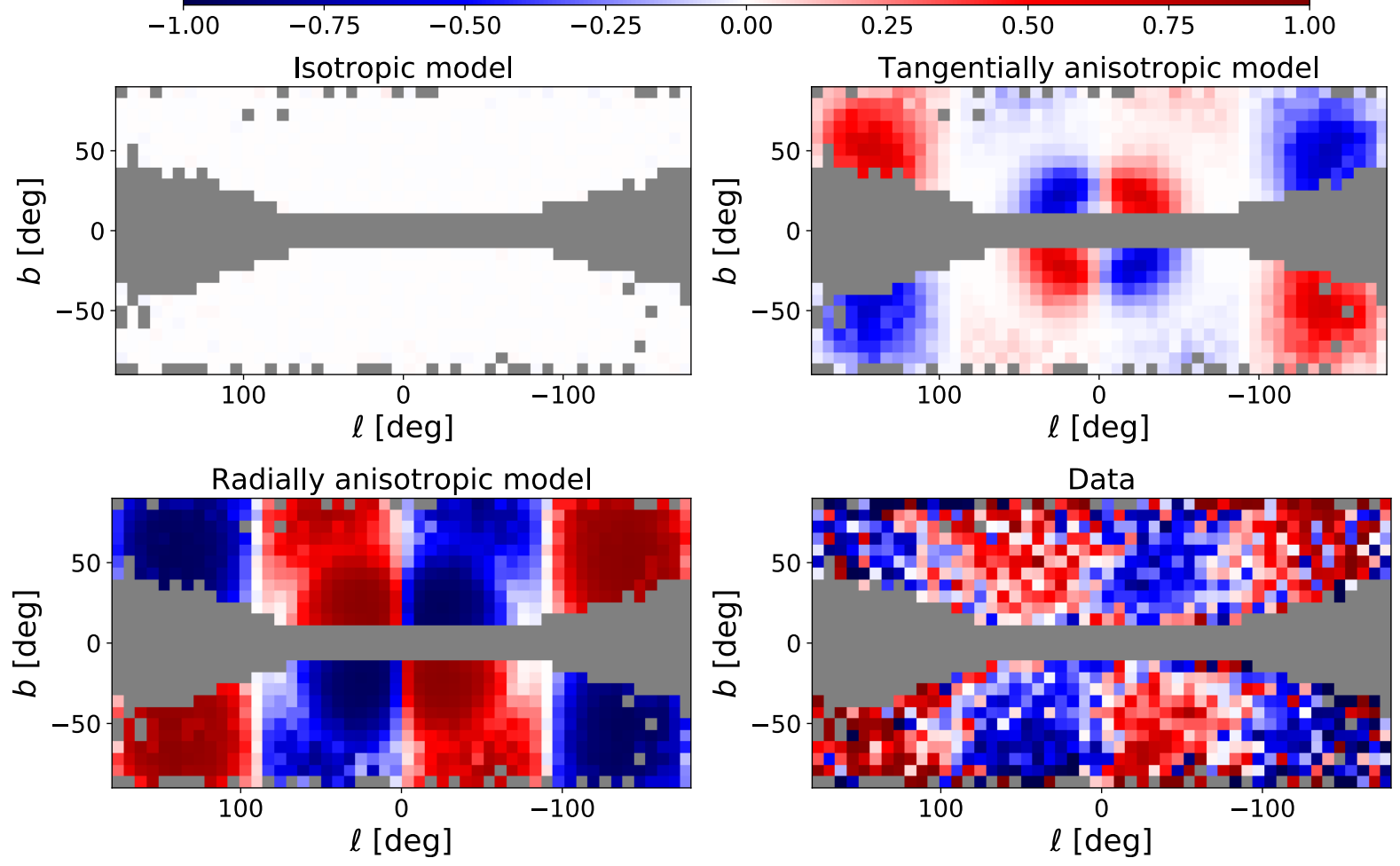

Figure 5. Pearson correlation coefficient of the Galactic proper motion components $\left(\mu_{\ell}, \mu_{b}\right)$ as a function of the sky position in Galactic coordinates for the three models (isotropic, top-left panel; tangentially anisotropic, top-right panel; radially anisotropic, bottom-left panel and the data bottom-right panel). Only the stars in the clean sample with $5<|z / \mathrm{kpc}|<30$ and located within the black ellipse shown in the left-hand panel of Fig. $3(N \sim 21,000)$ are considered. The underlying model velocity distributions are shown in the top-left panel of Fig. 4. The maps of the model correlation have been obtained by averaging the results of 10,000 model realisations. The mean dispersion of the model realisations $\left(\sigma \rho_{\mu_{\ell}, \mu_{\mathrm{b}}} \sim 0.25\right)$ is compatible with the level of random fluctuations displayed by the data. The proper motions have been corrected for the Solar reflex motion. The gray regions indicate bins without stars.

\subsection{Radial trends}

This sub-section explores the dependence of the RRL kinematics on the Galactocentric distance. In particular, we replicate the analysis presented above using sub-samples of RRL in different Galactocentric distance $r$ bins. Given the (maximal) spatial blurring due to the uncertainties in the distance estimate (Sec. 3.1), the minimal size of a distance bin is $4 \mathrm{kpc}$. For each radial bin, we show both the 1-D distributions of the transverse velocities derived from the Gaia DR2 proper motions (Fig. 6) and the 2-D maps of the correlation coefficient of the RRL proper motions on the sky (Fig. 7).

Velocity distributions evolution. The amplitude of the proper motion depends both on the velocity of a star as well as its distance from the observer (Eq. 2). Therefore, we convert the proper motions into physical velocities $V_{\ell}=K \mu_{\ell} D_{\odot}$ and $V_{b}=K \mu_{b} D_{\odot}$ to track changes in kinematics across a wide range of $r$. Here, $V_{\ell}$ and $V_{b}$ are the velocities along the direction of the Galactic longitude and Galactic latitude and $K \approx 4.70$ is the conversion factor from mas kpc $\mathrm{yr}^{-1}$ to $\mathrm{km} \mathrm{s}^{-1}$. The distributions of $V_{\ell}$ and $V_{b}$ as a function of the Galactocentric distance are given in Fig. 6. In the bin closest to the Galactic center, the two distributions are almost identical, in agreement with the isotropic model (see the bottom-left panel in Fig. 4). At the intermediate radii $(4<r / \mathrm{kpc}<30)$, the $V_{\ell}$ and $V_{b}$ distributions become progressively narrower; moreover, significant differences between $V_{b}$ and $V_{\ell}$ histograms start to appear. Thus, the kinematics of the RRL stars in this radial range appears compatible with the expectation from the radially anisotropic model (see the bottom-right panel in Fig. 4). Beyond 30 $\mathrm{kpc}$, the trend is reversed: the $V_{\ell}$ and $V_{b}$ distributions become broader and barely distinguishable, in agreement with the isotropic model. Please note that some of the broadening in the last bin is driven by large velocity errors for these distant and faint stars $(G \gtrsim 18.8$, see the error-bars in the top-left corner of each panel). To illustrate the effect of error-blurring on the shape of the velocity histograms, we deconvolve the distributions using the ED technique (Bovy Jo et al. 2011). The resulting deconvolved distributions are shown as dashed lines in Fig. 6. In the first four bins, i.e. for $r<30 \mathrm{kpc}$, the difference between the observed and deconvolved distributions is minuscule. In the last two radial bins, the mismatch is more noticeable but still minor. We conclude that the change in the shape of the transverse velocity distribution with radius is dictated primarily by the change in the overall anisotropy of the stellar halo.

We have checked that the model distributions of the transverse velocities do not substantially change their behavior as a function of $r$. Therefore, we confirm that the radial evolution shown in Fig. 6 is not compatible with a 

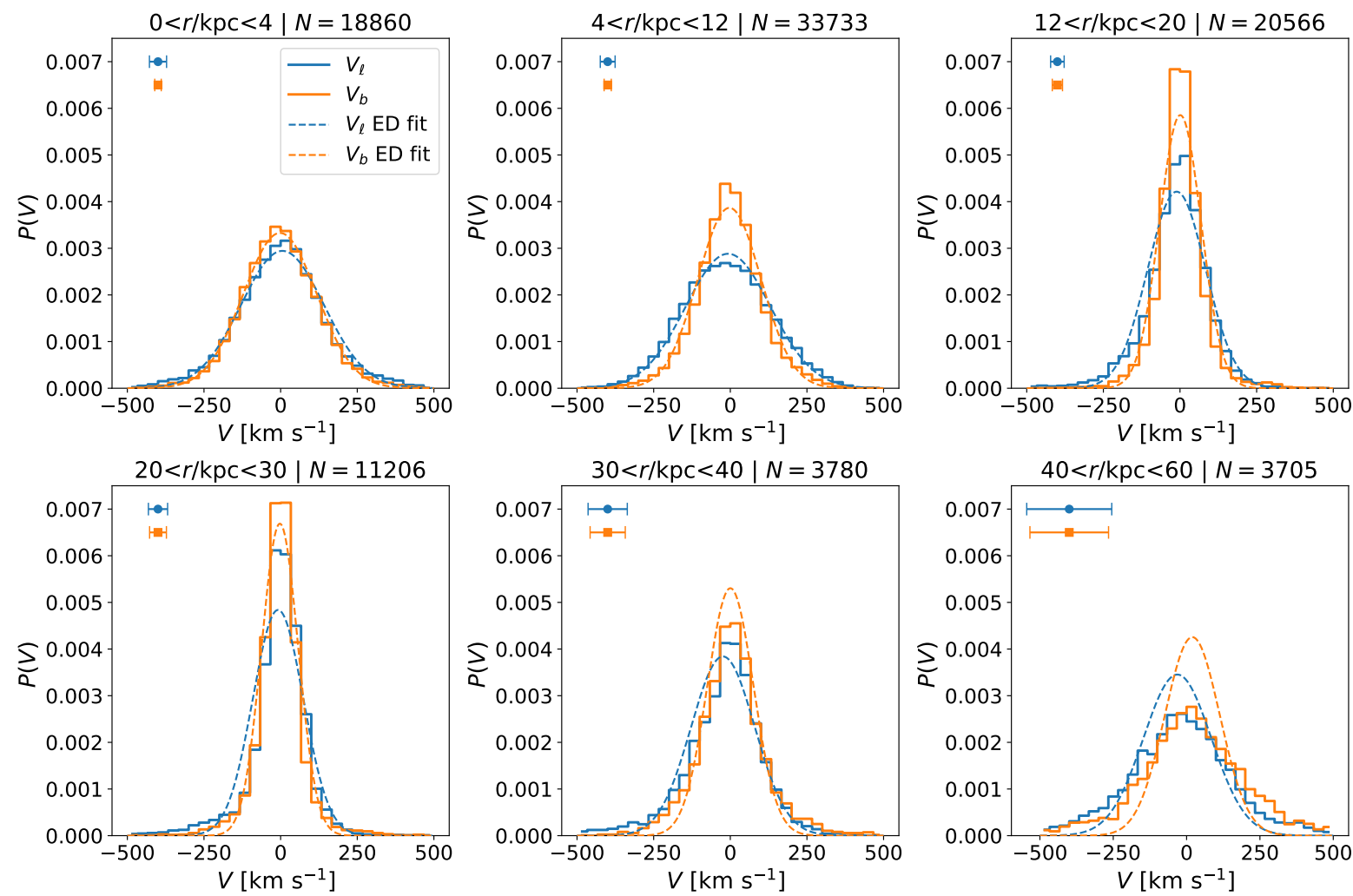

Figure 6. Distributions of the transverse velocities along the direction of the Galactic longitude $\left(V_{\ell}\right.$, blue histograms) and latitude $\left(V_{b}\right.$, orange histograms) for the RR Lyrae stars in the clean sample. The panels show the velocity distributions in different radial bins as reported in the title of each panel together with the number of stars $(N)$ in the bin. The velocities have been corrected for the Solar reflex motion (Schönrich 2012). The error bars in the top-left corners indicate the median velocity errors in each bin (blue-dot for $V_{\ell}$, orange-square for $V_{b}$ ). The velocity errors have been estimated by means of Monte Carlo simulations taking into account the observed proper motion errors and the estimated dispersion in the RR Lyrae absolute magnitude distribution (see Sec. 3.1). The dashed lines represent the results of Extreme Deconvolution Gaussian fits (Bovy Jo et al. 2011) taking into account the errors on $V_{\ell}$ and $V_{b}$.

single kinematic model. Instead, the data displays a rapid transition from isotropy to radial anisotropy at small radii around $4 \mathrm{kpc}$. There appears to be another transition, at larger distances, beyond $30 \mathrm{kpc}$, where even though the velocity errors grow substantially, the RRL motions resemble those generated by the isotropic model.

Correlation coefficient evolution. The change from the orbital isotropy to the radial anisotropy (and back) is not only apparent in the amplitudes of the transversal velocities shown in Fig. 6 but it is also reflected in the directions of RRL motions displayed in Fig. 7. The Figure reproduces the map shown in the bottom-right panel of Fig. 5 but now as a function of the Galactocentric distance. The red-blue pattern typical of the streaming along the radial direction (see the bottom-left panel in Fig. 5) is already noticeable in the innermost bin, albeit with a small amplitude. Between 4 and $20 \mathrm{kpc}$ this pattern becomes much more pronounced (see the bottom-right panel of Fig. 5 for comparison). Outside of $20 \mathrm{kpc}$, especially in the last two bins, the correlation coefficient maps lose their coherence quickly, displaying a mix of random orientations. The patterns at higher distances should be interpreted with caution, as the growing velocity errors in these bins will act to destroy intrinsic correlations, especially the low-amplitude ones.

In conclusion, Fig. 6 and Fig. 7 confirm that between
4 and 20-30 kpc from the Galactic centre most of the stars have proper motions compatible with a model with a strong radial anisotropy. Outside of this region, the distribution of proper motions appears to behave similar to the isotropic or, perhaps much less radially biased, model. This kind of radial trend is expected if the inner halo is dominated by highly eccentric orbits $(e>0.8)$. In this case, along most of the orbital path, the star's velocity is predominantly in the radial component. However, close to the turn-around points, namely the pericentre and the apocentre, the radial component of the velocity rapidly decreases to 0 . Of course, the synchronized change in the orbital anisotropy in large portions of the halo is only possible if the halo is dominated by the tidal debris from a single accretion event. In this case, the stars naturally possess similar orbital properties, thus causing the whole halo's anisotropy to change quickly around their pericentres and apocentres.

Our finding of the switch in the orbital properties of the stellar halo around $4 \mathrm{kpc}$ is in good agreement with the detection of the anisotropy change at similar distances presented in Wegg et al. (2018) and with the estimate of the pericenters of the stars belonging to the Hercules-Aquila and Virgo Clouds in Simion et al. (2018). The decay of the correlation between the proper motion components beyond 30 $\mathrm{kpc}$ is in agreement with the marked anisotropy decrease 


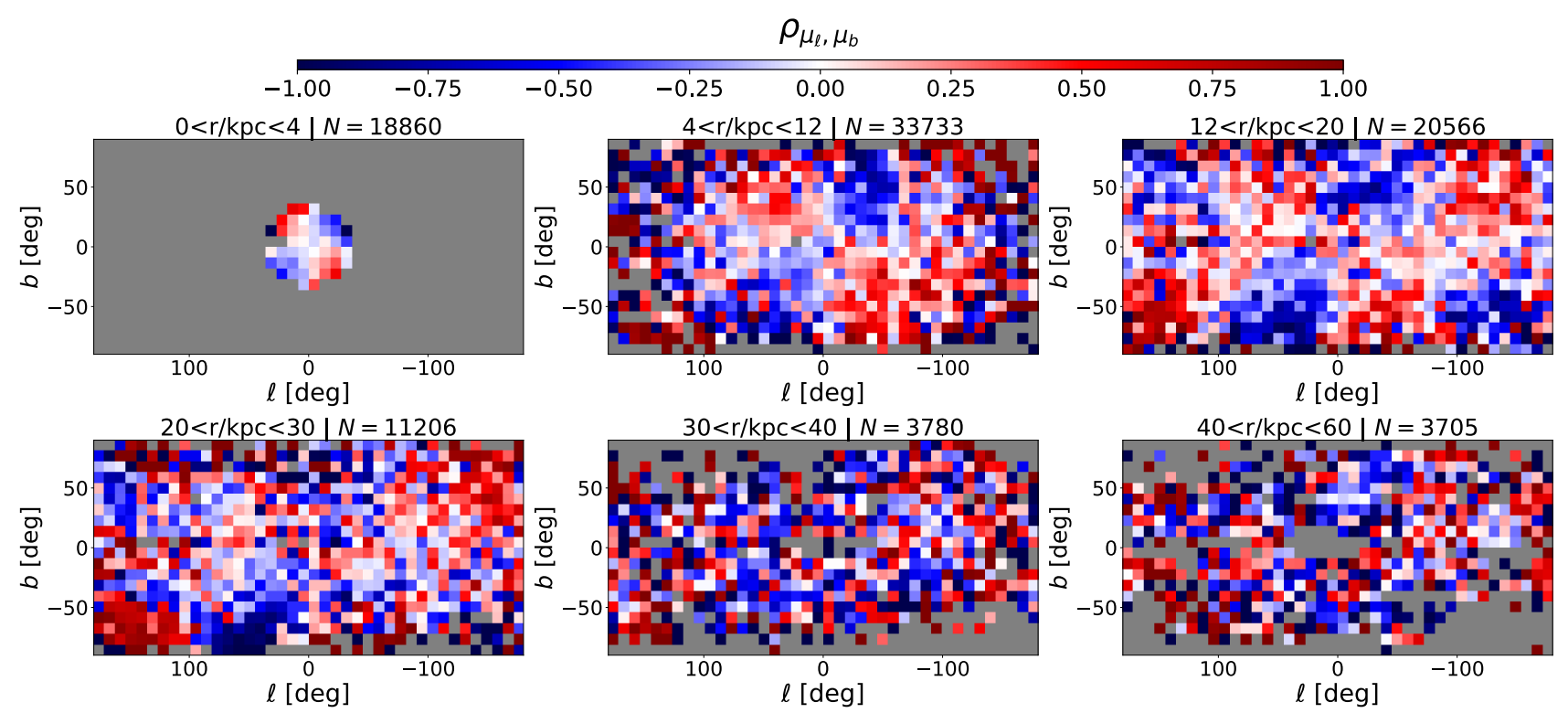

Figure 7. Same as Fig. 5, but considering all RR Lyrae stars in the clean sample in different radials bins as reported in the title of each panel together with the number of stars in the bin. The gray regions indicate bins without stars. Note that the strongest negative correlation (dark blue color) in the fourth radial bin $(20-30 \mathrm{kpc})$ is supplied by the remaining portion of the Sgr trailing stream.

measured at similar distances by Bird et al. (2018) and Lancaster et al. (2018). This behavior can be explained by the traversal of the apocentric pile-up of a large number of stars sharing a common progenitor as described in Deason et al. (2018). Moreover, the evolution of the RRL orbital anisotropy with Galactocentric distance appears to correlate well with the change in the mixture of RRL periods and amplitudes (see Belokurov et al. 2018a). Together with these earlier works, out study gives further support to the hypothesis of Deason et al. (2013) in which the observed stellar halo density break can be linked to the last apocentre of a massive progenitor galaxy, whose tidal debris engulfed the inner Milky Way.

\subsection{Signature of an ancient major merger?}

The simple analysis presented in this Section suggests that the orbits of the bulk of the RRL stars in the inner halo of the Milky Way (including the HAC and the VOD) are highly eccentric, in line with the local measurements of the "Gaia Sausage"(see Belokurov et al. 2018b; Myeong et al. 2018b; Helmi et al. 2018).

The exact fraction of RRL stars in our sample that can be associated with this structure is uncertain. Recent studies have shown that the fraction of the inner halo population with strong anisotropy is certainly larger than 50\% (Lancaster et al. 2018) and can be as high as 70\% (Mackereth et al. 2018). We repeated the analysis described in Sec. 4.1 considering a mixed model with both isotropic and strong radially anisotropic velocity distributions. We found that the magnitude of the red-blue patterns shown by the data in Fig. 5 are qualitatively reproduced when the fraction of stars belonging to the anisotropic population is larger than $70 \%$. Interestingly, several works have shown that, during mergers, large numbers of stars in the pre-existing disc can be heated to more eccentric and energetic orbits form- ing an in-situ population in the inner halo (e.g. Purcell et al. 2010; Font et al. 2011). Although the kinematic properties of this kicked-up population may resemble those of the accreted debris (see e.g. Di Matteo et al. 2011; Jean-Baptiste et al. 2017), the in-situ stars tend to maintain a good fraction of their initial (positive) $z$-angular momentum (see e.g. Haywood et al. 2018). Since the z-angular momentum of the "Gaia Sausage" stars is typically very low or negative (see Belokurov et al. 2018b; Myeong et al. 2018b; Helmi et al. 2018), we envisage that the contribution of the heated disc stars to our sample is likely minimal. We therefore conclude that the triaxial structure uncovered in Sec. 3 is mostly composed of the tidal debris from this ancient massive merger. While glimpses of the "Gaia Sausage" have recently been found outside of the Solar neighborhood (see Deason et al. 2018; Lancaster et al. 2018; Simion et al. 2018), ours is the first comprehensive 3-D map of this gigantic debris structure which completely dominates the inner stellar halo.

There exists an additional corollary worth pondering about. Depending on the exact time of accretion and the mass of the progenitor galaxy, the "Gaia Sausage" might also be contributing a significant fraction of the Dark Matter content in the inner part of the Milky Way. If the merger delivered some $10^{11} M_{\odot}$ of Dark Matter - which is not far off most currently available estimates (see Belokurov et al. 2018b; Helmi et al. 2018; Mackereth et al. 2018) - then its portion of the local Dark Matter budget can be gauged as follows. Using the above progenitor mass and the triaxial model described in the previous Section, the Dark Matter density at the Solar (elliptical) radius due to the "Gaia Sausage" is $\sim 7 \times 10^{-4} \mathrm{M}_{\odot} \mathrm{pc}^{-3}$. This is approximately $10 \%$ of the local Dark Matter density (see Bovy \& Tremaine 2012). Quite probably, however, this is a lower bound as the density in the central parts of the debris cloud is over-estimated by our parametric model (see the left-hand panel of Fig. 3). Naturally, we expect a depletion in density at distances inward of 
the pericentre. A different estimate can be obtained by noticing that within $30 \mathrm{kpc}$, i.e. inside the apocentre of the "Gaia Sausage", the MW mass is estimated to be $\sim 2.5 \times 10^{11} \mathrm{M}_{\odot}$ (see e.g. Gibbons et al. 2014; Williams et al. 2017; Posti \& Helmi 2018), of which at least $0.5 \times 10^{11} \mathrm{M}_{\odot}$ can be attributed to the baryonic component (see McMillan 2017). Therefore, the Dark Matter contribution associated with the principal component of the stellar halo can be $\sim 50 \%$ within $30 \mathrm{kpc}$.

In this regard, it is curious that the direction of the major axis of the triaxial stellar halo described in this work is close (within $20^{\circ}$ ) to the direction of the major axis of the triaxial Dark Matter halo model found by Law et al. (2009). Although the details of the axial ratios show some difference (the Dark Matter shortest axis is not on the direction normal to the Galactic plane), the "amount" of triaxiality as measured by the triaxiality parameter $T^{3}$ is very similar. While the exact orientation of the Dark Matter halo with respect to the Galactic disk as suggested by Law et al. (2009) is not stable, the triaxial Dark Matter halo and the baryonic disk can co-exist happily if the disk's spin is allowed to be misaligned with respect to the principal planes of the potential (see Debattista et al. 2013). This misalignment can be induced, for example, by gas accretion at later times as demonstrated in Debattista et al. (2015).

\section{CONCLUSIONS}

In this paper, we take advantage of the most comprehensive sample of RRL stars to date, i.e. the one supplied by the Gaia DR2, to study the shape of the Galactic stellar halo. In order to assemble a dataset with sensible and stable completeness and purity, we had to get rid of more than a half of the original total of $\sim 230,000$ objects. While this may sound scary, remember that the estimated contamination of the Gaia DR2 sample shoots up to 50\% in the bulge area (see Holl et al. 2018). For our investigations, we have only kept RRL stars that possess well-behaved colors and astrometry. Additionally, we do not consider objects residing in small-scale over-densities, i.e. known Milky Way satellites and globular clusters. However, even after all these cuts, we are left with nearly 93,000 RRL stars across the entire sky, from the inner several kpc to the distant outskirts - a dataset unprecedented in its richness and reach.

For the very first time, we produce detailed maps of the RRL density distribution in the inner Milky Way (see Fig. 2). Slicing the Galaxy at different Galactic heights, we reveal the evolution of the halo shape from nearly spherical (close to the Galaxy's centre) to clearly triaxial further out. Despite several obvious asymmetries and large-scale overdensities visible in these maps, the overall structure of the halo appears to be well-described by the SPL-TR ${ }^{q v}$ model of Iorio et al. (2018). In this model, the major axis lies in the Galactic plane rotated by $\sim 70^{\circ}$ (anti-clockwise) away from the Galactic $x$-axis, i.e. pointing approximately in the direction of the Magellanic Clouds. The ratio of the major to intermediate axis is $p=1.3$, but the ratio of the minor axis (aligned with the Galactic $z$ axis) to major one changes

$3 T=\frac{1-b^{2} / a^{2}}{1-c^{2} / a^{2}}$, where $a, b, c$ are the longest, intermediate and shortest axes, respectively. with distance from the Milky Way centre (from $q \sim 0.5$ to $q \sim$ 0.8 ). The clearest deviations from the above model are the Virgo Over-Density and the Hercules-Aquila Cloud, which protrude far in the vertical direction on the opposite sides of the Galactic plane. As we have demonstrated in Section 3.2 and Fig. 3, the SPL-TR ${ }^{q v}$ model of Iorio et al. (2018) can be adjusted slightly to encompass both HAC and VOD. This is achieved by bringing its intermediate axis out of the Galactic plane by $\sim 20^{\circ}$. From the slices presented in Fig. 2 it is now easy to judge the whereabouts of the HAC and VOD with respect to the Milky Way's centre and to each other. These debris clouds appear to be aligned with the major axis of the triaxial structure discussed above.

The first clues that HAC and VOD may actually be part of the same accretion event have already been presented by Simion et al. (2018), who calculated the orbital properties of a sub-sample of RRL stars residing in these over-densities. Given that both structures appear to be dominated by stars on extremely eccentric orbits, Simion et al. (2018) conclude that HAC and VOD represent unmixed portions of an ancient massive head-on collision also known as the "Gaia Sausage". In an attempt to test this hypothesis, we have studied the kinematics of the inner halo RRL stars as reflected in their proper motions. We claim that across a wide range of distances from the Sun, the halo's velocity ellipsoid can be gleaned from its projection on the celestial sphere. Our results are in good agreement with an earlier analysis of the RRL proper motion data of Wegg et al. (2018). Compared to the above study, while we do not attempt to model the shape of the velocity ellipsoid, we do obtain a broader view of the global kinematic patterns in the Galactic halo. Accordingly, contrasting the observed amplitudes and directions of the RRL proper motions within $\sim 30$ kpc from the Galactic centre with simple kinematic models, we show that the entire inner halo is dominated by stars on highly eccentric orbits.

We interpret the stretched appearance and the extreme radial anisotropy of the inner halo as the tell-tale signs of a low angular momentum collision with a massive satellite (see e.g. Brook et al. 2003), thus associating the bulk of the RRL stars within $30 \mathrm{kpc}$ with the so-called "Gaia Sausage" merger event (Belokurov et al. 2018b; Myeong et al. 2018b; Haywood et al. 2018; Helmi et al. 2018). By tracking the change in the behavior of the RRL proper motions with Galactocentric distance, we place constraints on the pericentre $(\lesssim 4 \mathrm{kpc})$ and the apocentre $(20-40 \mathrm{kpc})$ of this enormous tidal debris cloud. The fact that today it appears to be squashed vertically (in the direction perpendicular to the Galactic disc) agrees well with the predictions of numerical simulations of the Dark Matter halo evolution in the presence of baryons (Kazantzidis et al. 2004; Gnedin et al. 2004; Debattista et al. 2008; Abadi et al. 2010). In terms of local Dark Matter density, we have estimated the contribution of the "Gaia Sausage" to be between $10 \%$ and 50\%. A curious conundrum is starting to emerge in which a clearly triaxial stellar halo needs to be reconciled with multiple recent reports of a near spherical inner Dark Matter halo (Bowden et al. 2015; Bovy et al. 2016; Wegg et al. 2018), a measurement itself in tension with the results from numerical simulations which do not produce perfect sphericity (e.g. Kazantzidis et al. 2010; Debattista et al. 2015). The spatio-kinematic information uncovered here will help con- 
strain the total mass as well as the time of accretion of the "Gaia Sausage" progenitor, and thus understand the role this event played in the life of the Milky Way.

\section{ACKNOWLEDGEMENTS}

The authors thank Wyn Evans, Victor Debattista, Andrew Cooper, Sergey Koposov, Iulia Simion, Alis Deason for most illuminating discussions which helped to improve this manuscript. G.I. is supported by the Royal Society Newton International Fellowship. The research leading to these results has received funding from the European Research Council under the European Union's Seventh Framework Programme (FP/2007-2013) / ERC Grant Agreement n. 308024. This work has made use of data from the European Space Agency (ESA) mission Gaia (https://www. cosmos.esa.int/gaia), processed by the Gaia Data Processing and Analysis Consortium (DPAC, https://www. cosmos.esa.int/web/gaia/dpac/consortium). Funding for the DPAC has been provided by national institutions, in particular the institutions participating in the Gaia Multilateral Agreement.

\section{REFERENCES}

Abadi M. G., Navarro J. F., Fardal M., Babul A., Steinmetz M., 2010, MNRAS, 407, 435

Alam S., et al., 2015, ApJS, 219, 12

Allgood B., Flores R. A., Primack J. R., Kravtsov A. V., Wechsler R. H., Faltenbacher A., Bullock J. S., 2006, MNRAS, 367, 1781

Altay G., Colberg J. M., Croft R. A. C., 2006, MNRAS, 370, 1422 Avila-Reese V., Colín P., Valenzuela O., D’Onghia E., Firmani C., 2001, ApJ, 559, 516

Bailer-Jones C. A. L., Rybizki J., Fouesneau M., Mantelet G., Andrae R., 2018, preprint, (arXiv:1804.10121)

Bell E. F., et al., 2008, ApJ, 680, 295

Belokurov V., et al., 2007, ApJ, 657, L89

Belokurov V., et al., 2014, MNRAS, 437, 116

Belokurov V., Erkal D., Deason A. J., Koposov S. E., De Angeli F., Evans D. W., Fraternali F., Mackey D., 2017, MNRAS, 466,4711

Belokurov V., Deason A. J., Koposov S. E., Catelan M., Erkal D., Drake A. J., Evans N. W., 2018a, MNRAS, 477, 1472

Belokurov V., Erkal D., Evans N. W., Koposov S. E., Deason A. J., 2018b, MNRAS, 478, 611

Benson A. J., Lacey C. G., Frenk C. S., Baugh C. M., Cole S., 2004, MNRAS, 351, 1215

Bett P., Eke V., Frenk C. S., Jenkins A., Helly J., Navarro J., 2007, MNRAS, 376, 215

Bird S. A., Xue X.-X., Liu C., Shen J., Flynn C., Yang C., 2018, preprint, (arXiv:1805.04503)

Bonaca A., et al., 2012, AJ, 143, 105

Bovy J., Tremaine S., 2012, ApJ, 756, 89

Bovy Jo Hogg D. W., Roweis S. T., 2011, Annals of Applied Statistics, 5

Bovy J., Bahmanyar A., Fritz T. K., Kallivayalil N., 2016, ApJ, 833, 31

Bowden A., Belokurov V., Evans N. W., 2015, MNRAS, 449, 1391

Brook C. B., Kawata D., Gibson B. K., Flynn C., 2003, ApJ, 585, L125

Bryan S. E., Kay S. T., Duffy A. R., Schaye J., Dalla Vecchia C., Booth C. M., 2013, MNRAS, 429, 3316

Bullock J. S., Johnston K. V., 2005, ApJ, 635, 931
Carollo D., et al., 2007, Nature, 450, 1020

Carollo D., et al., 2010, ApJ, 712, 692

Catelan M., Pritzl B. J., Smith H. A., 2004, ApJS, 154, 633

Clementini G., et al., 2018, preprint, (arXiv:1805.02079)

Colberg J. M., White S. D. M., Jenkins A., Pearce F. R., 1999, MNRAS, 308, 593

Cole S., Lacey C., 1996, MNRAS, 281, 716

Cooper A. P., et al., 2010, MNRAS, 406, 744

Cooper A. P., Parry O. H., Lowing B., Cole S., Frenk C., 2015, MNRAS, 454, 3185

D'Souza R., Bell E. F., 2018, MNRAS, 474, 5300

Davé R., Spergel D. N., Steinhardt P. J., Wandelt B. D., 2001, ApJ, 547, 574

De Lucia G., Helmi A., 2008, MNRAS, 391, 14

Deason A. J., Belokurov V., Evans N. W., 2011, MNRAS, 416, 2903

Deason A. J., Belokurov V., Evans N. W., Johnston K. V., 2013, ApJ, 763, 113

Deason A. J., Mao Y.-Y., Wechsler R. H., 2016, ApJ, 821, 5

Deason A. J., Belokurov V., Koposov S. E., Lancaster L., 2018, ApJ, 862, L1

Debattista V. P., Moore B., Quinn T., Kazantzidis S., Maas R., Mayer L., Read J., Stadel J., 2008, ApJ, 681, 1076

Debattista V. P., Roškar R., Valluri M., Quinn T., Moore B., Wadsley J., 2013, MNRAS, 434, 2971

Debattista V. P., van den Bosch F. C., Roškar R., Quinn T., Moore B., Cole D. R., 2015, MNRAS, 452, 4094

Di Matteo P., Lehnert M. D., Qu Y., van Driel W., 2011, A\&A, 525, L3

Dubinski J., Carlberg R. G., 1991, ApJ, 378, 496

Duffau S., Zinn R., Vivas A. K., Carraro G., Méndez R. A., Winnick R., Gallart C., 2006, ApJ, 636, L97

Evans D. W., et al., 2018, preprint, (arXiv:1804.09368)

Font A. S., McCarthy I. G., Crain R. A., Theuns T., Schaye J., Wiersma R. P. C., Dalla Vecchia C., 2011, MNRAS, 416, 2802

Frenk C. S., White S. D. M., Davis M., Efstathiou G., 1988, ApJ, 327,507

Gaia Collaboration et al., 2016, A\&A, 595, A1

Gaia Collaboration et al., 2018b, preprint, (arXiv:1804.09381)

Gaia Collaboration Brown A. G. A., Vallenari A., Prusti T., de Bruijne J. H. J., Babusiaux C., Bailer-Jones C. A. L., 2018a, preprint, (arXiv:1804.09365)

Gibbons S. L. J., Belokurov V., Evans N. W., 2014, MNRAS, 445, 3788

Gnedin O. Y., Kravtsov A. V., Klypin A. A., Nagai D., 2004, ApJ, 616, 16

Hahn O., Porciani C., Carollo C. M., Dekel A., 2007, MNRAS, 375,489

Harris W. E., 2010, preprint, (arXiv:1012.3224)

Hayashi E., Navarro J. F., Springel V., 2007, MNRAS, 377, 50

Hayes C. R., et al., 2018, ApJ, 852, 49

Haywood M., Di Matteo P., Lehnert M., Snaith O., Khoperskov S., Gómez A., 2018, preprint, (arXiv:1805.02617)

Helmi A., Babusiaux C., Koppelman H. H., Massari D., Veljanoski J., Brown A. G. A., 2018, preprint, (arXiv:1806.06038)

Holl B., et al., 2018, preprint, (arXiv:1804.09373)

Iorio G., Belokurov V., Erkal D., Koposov S. E., Nipoti C., Fraternali F., 2018, MNRAS, 474, 2142

Jean-Baptiste I., Di Matteo P., Haywood M., Gómez A., Montuori M., Combes F., Semelin B., 2017, A\&A, 604, A106

Jurić M., et al., 2008, ApJ, 673, 864

Kafle P. R., Sharma S., Lewis G. F., Bland-Hawthorn J., 2012, ApJ, 761, 98

Kazantzidis S., Kravtsov A. V., Zentner A. R., Allgood B., Nagai D., Moore B., 2004, ApJ, 611, L73

Kazantzidis S., Abadi M. G., Navarro J. F., 2010, ApJ, 720, L62

King III C., Brown W. R., Geller M. J., Kenyon S. J., 2015, ApJ, 813,89 
Koposov S. E., Belokurov V., Torrealba G., 2017, MNRAS, 470, 2702

Kruijssen J. M. D., Pfeffer J. L., Reina-Campos M., Crain R. A., Bastian N., 2018, MNRAS,

Lancaster L., Koposov S. E., Belokurov V., Evans N. W., Deason A. J., 2018, preprint, (arXiv:1807.04290)

Law D. R., Majewski S. R., Johnston K. V., 2009, ApJ, 703, L67

Libeskind N. I., Hoffman Y., Forero-Romero J., Gottlöber S., Knebe A., Steinmetz M., Klypin A., 2013, MNRAS, 428, 2489

Lindegren L., Lammers U., Hobbs D., O'Mullane W., Bastian U., Hernández J., 2012, A\&A, 538, A78

Lindegren L., et al., 2018, preprint, (arXiv:1804.09366)

Mackereth J. T., et al., 2018, preprint, (arXiv:1808.00968)

Mayer L., Moore B., Quinn T., Governato F., Stadel J., 2002, MNRAS, 336, 119

McCarthy I. G., Font A. S., Crain R. A., Deason A. J., Schaye J., Theuns T., 2012, MNRAS, 420, 2245

McMillan P. J., 2017, MNRAS, 465, 76

Monachesi A., et al., 2018, preprint, (arXiv:1804.07798)

Moore B., Kazantzidis S., Diemand J., Stadel J., 2004, MNRAS, 354,522

Morrison H. L., Mateo M., Olszewski E. W., Harding P., DohmPalmer R. C., Freeman K. C., Norris J. E., Morita M., 2000, AJ, 119, 2254

Muraveva T., Delgado H. E., Clementini G., Sarro L. M., Garofalo A., 2018, preprint, (arXiv:1805.08742)

Myeong G. C., Evans N. W., Belokurov V., Sanders J. L., Koposov S. E., 2018a, preprint, (arXiv:1805.00453)

Myeong G. C., Evans N. W., Belokurov V., Sanders J. L., Koposov S. E., 2018b, ApJ, 856, L26

Necib L., Lisanti M., Belokurov V., 2018, preprint, (arXiv: 1807.02519 )

Newberg H. J., Yanny B., 2006, in Journal of Physics Conference Series. pp 195-204 (arXiv:astro-ph/0507671), doi:10.1088/1742-6596/47/1/024

Newberg H. J., et al., 2002, ApJ, 569, 245

Peter A. H. G., Rocha M., Bullock J. S., Kaplinghat M., 2013, MNRAS, 430, 105

Piersimoni A. M., Bono G., Ripepi V., 2002, AJ, 124, 1528

Posti L., Helmi A., 2018, preprint, (arXiv: 1805.01408)

Preston G. W., Shectman S. A., Beers T. C., 1991, ApJ, 375, 121

Purcell C. W., Bullock J. S., Kazantzidis S., 2010, MNRAS, 404, 1711

Reid N., Majewski S. R., 1993, ApJ, 409, 635

Schlegel D. J., Finkbeiner D. P., Davis M., 1998, ApJ, 500, 525

Schönrich R., 2012, MNRAS, 427, 274

Sesar B., Jurić M., Ivezić Ž., 2011, ApJ, 731, 4

Siegel M. H., Majewski S. R., Reid I. N., Thompson I. B., 2002, ApJ, 578, 151

Simion I. T., Belokurov V., Irwin M., Koposov S. E., 2014, MNRAS, 440, 161

Simion I. T., Belokurov V., Koposov S. E., 2018, preprint, (arXiv: 1807.01335 )

Sluis A. P. N., Arnold R. A., 1998, MNRAS, 297, 732

Tissera P. B., Scannapieco C., Beers T. C., Carollo D., 2013, MNRAS, 432, 3391

Tomassetti M., et al., 2016, MNRAS, 458, 4477

Tormen G., 1997, MNRAS, 290, 411

Vera-Ciro C. A., Sales L. V., Helmi A., Frenk C. S., Navarro J. F., Springel V., Vogelsberger M., White S. D. M., 2011, MNRAS, 416, 1377

Vivas A. K., et al., 2001, ApJ, 554, L33

Warren M. S., Quinn P. J., Salmon J. K., Zurek W. H., 1992, ApJ, 399, 405

Wegg C., Gerhard O., Bieth M., 2018, preprint, (arXiv: 1806.09635)

Williams A. A., Belokurov V., Casey A. R., Evans N. W., 2017, MNRAS, 468, 2359
Xue X.-X., Rix H.-W., Ma Z., Morrison H., Bovy J., Sesar B., Janesh W., 2015, ApJ, 809, 144

Zolotov A., Willman B., Brooks A. M., Governato F., Brook C. B., Hogg D. W., Quinn T., Stinson G., 2009, ApJ, 702, 1058 van der Marel R. P., Sahlmann J., 2016, ApJ, 832, L23

This paper has been typeset from a $\mathrm{T}_{\mathrm{E}} \mathrm{X} / \mathrm{L}_{\mathrm{A}} \mathrm{T} \mathrm{E}$ file prepared by the author. 